\title{
Interference Issuses of Radio Frequency Identification Devices in Magnetic Resonance Imaging Systems and Computed Tomography Scan
}

\author{
M. Periyasamy ${ }^{1 *}$ and R. Dhanasekaran ${ }^{2}$ \\ ${ }^{1}$ Professor, Syed Ammal Engineering College, Ramanathapuram, India \\ ${ }^{2}$ Director-Research, Syed Ammal Engineering College, Ramanathapuram, India
}

(Received 7 May 2015, Received in final form 11 August 2015, Accepted 11 August 2015)

\begin{abstract}
We evaluated certain issues related to magnetic resonance imaging (MRI) coupled with the use of active 2.5 GHz radio frequency identification (RFID) tags for patient identification using low field (0.3 T) MRI and computed tomography (CT) scans. We also investigated the performance of the RFID reader located outside the MRI room by considering several factors. A total of ten active RFID tags were exposed to several MRI sequences and X-rays of CT scan. We found that only card type active RFID tags are suitable for patient identification purpose in MRI environment and both wristbands as well as card tags were suitable for the same in CT environment. Severe artifacts were found in the captured MRI and CT images when the area of the imaging was in proximity to the tags. No external factors affected the performance of active RFID reader stationed outside the MRI scan room.
\end{abstract}

Keywords : active radio frequency identification, artifacts, electromagnetic interference, magnetic resonance imaging, magnetic susceptibility, patient safety

\section{Introduction}

Currently, magnetic resonance imaging (MRI) is widely used in India as well as in other countries [1] for diagnosing anomalies and disorders in human bodies. Since MRI emits strong electromagnetic (EM) waves, patients having certain implants/bio-medical devices with metallic parts or magnetically-activated devices/programmable devices were contraindicated for MRI environment [2-4]. Such devices or implants are harmful not only to the patients carrying them but also to the functionality of those devices or implants. They also introduce artifacts in the captured images that hamper proper diagnosis. Therefore, MRI operators should perform a pre-examination check of the patients to ensure that the patients have no such contraindicated implants/devices [5].

In the healthcare sector, accurate identification of the patient is a critical issue [6] and any shortfall in this category may result in irrevocable or irreparable loss to the patients, particularly in the case of surgeries [7-9]. Careful attention must be given to the accurate identification

(C)The Korean Magnetics Society. All rights reserved.

*Corresponding author: Tel: +91-4567-304103

Fax:+91-4567-304123, e-mail: peri_samym@yahoo.com of the patients during their stay in hospital. The adoption of suitable information technology (IT) tools can prevent many medical errors [10]. Of the several technologies available, radio frequency identification (RFID) has been gaining significant attention during the last decade for several reasons [11, 12]. RFID is a wireless technology in which the wristbands and transponders (tags) are attached to the object or person to be tracked. Each RFID tag contains a microchip (transceiver) connected to an antenna that can transmit/receive signals from/to reader. By exploiting the capabilities of RFID, hospitals can deliver better and safer patient care at a lower cost [13].

In order to ascertain the accurate identification of the patient during their hospital stay, they should be required to carry RFID wristband tags [14] that cannot be removed till they leave the hospital. Patients carrying wristband tags may require undergoing MRI or computed tomography (CT) examination for diagnostic purposes. Under these circumstances, a large amount of EM radiations from the MRI scanner or high-intensity X-rays of the $\mathrm{CT}$ scan can damage the tiny radio transceiver of RFID tags as well as its memory contents [15]. This may lead to the alteration of the device's functionality or deactivation of the tags. Additionally, devices such as RFID tags may also induce artifacts in the MRI images that can hamper the proper 
diagnosis by doctor [16]. Thus, it is important to study the effects of EM interactions between RFID tags and MRI scanner before implementing them in real time.

Literature $[15,16,17-20]$ related to the use of RFID in MRI environment have indicated that it was safe to use passive RFID tags or passive RFID implants operating at certain frequencies in several MRI environments. None of these studies reported the malfunction of the device except one where one of the tested implants got deactivated [18]. Most studies revealed that RFID tags induced artifacts in MRI images except in two investigations [28, 29]. Thus, these facts clearly indicate that there is the need to characterize every electronically-activated device/implant with regard to its use in each MRI environment. In the case of the CT examination, the CT scans do not affect the safety of the patients [15]. However, they may introduce artifacts in the images when the area of imaging is closer to the RFID tags. To the best of the author's knowledge, only one study [21] has discussed the usage of active $2.4 \mathrm{GHz}$ RFID tags (card tags) in an MRI environment of $1.5 \mathrm{~T}$, and concluded that tested tags were safe with no change in their functionality and induced severe artifacts in captured MRI images if the area of imaging is closer to tags. Since these results cannot be generalized to other MRI scanners with different field strengths, because of the difference in the design of MRI scanners by various manufacturers and complexity involved in the EM field interactions [24]. Thus, we decided to conduct similar tests with low field 0.3 T MRI scan using more number of sequences and different types of tags. Functional alteration and imaging artifacts are the two major issues in the utility of RFID tags in MRI environments $[28,29]$. Thus, the objective of the proposed work is to conduct invitro and invivo tests to evaluate the functional and imaging artifacts issues related to use of active $2.5 \mathrm{GHz}$ RFID tags in $0.3 \mathrm{~T}$ MRI scanners and CT scan. In addition to this, the proposed work will also evaluate the performance of RFID reader located outside the MRI room by considering several factors, including on/off conditions of the scanner, angle of reader, and the type of tags used.

\section{Materials}

\subsection{RFID System}

An RFID system contains three important components: reader (interrogator), transponder (tag), and the host computer. With the help of the software in the host computer, a reader can encode (write) or decode (read) the data in the transponders through a wireless medium. A schematic diagram of an active RFID system is given

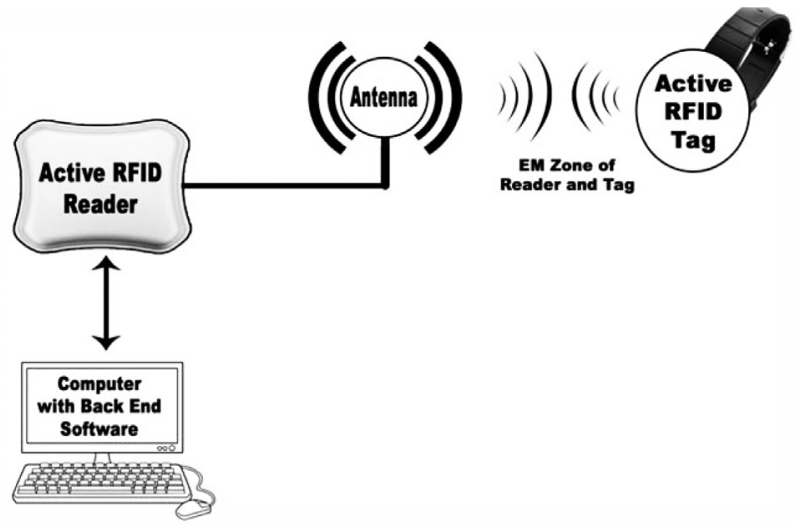

Fig. 1. Schematic diagram of active RFID System.

in Fig. 1. Each tag holds a unique identification number (UID) of several bytes length depending on the manufacturer. The tags can be broadly classified as passive and active. Passive tags are activated by EM signals emitted from the reader antenna and scattered back to the reader. In contrast, active tags are activated by its internal battery. RFID systems can operate in four different frequency bands such as low frequency (LF, 120-135 KHz), high frequency (HF, $13.56 \mathrm{MHz}$ ), ultrahigh frequency (UHF, $860-900 \mathrm{MHz})$ and microwave frequency $(2.4 \mathrm{GHz})$. Passive systems operate in LF, HF and UHF range while most of the active RFID systems operate in UHF and microwave frequencies [22].

In this proposed work, an active RFID system working at $2.4 \mathrm{GHz}$ is used that is supplied by the Spectrum Group Private Limited, Mumbai. The technical details of both the reader and the tags are summarized in Tables 1 and 2, respectively. The antennae of the tags are made up of copper material, which is considered as a paramagnetic, and the batteries are made up of nickel, which is considered as a ferromagnetic. The reader is of desktop type and can be fixed at any point.

Table 1. Technical specification of active RFID reader used in this work.

\begin{tabular}{ll}
\hline \hline Parameter & Specifications \\
\hline Operating Frequency & $2.4-2.8 \mathrm{GHz}$ \\
Maximum Simultaneous tag detection & 200 tags \\
Communication Standard & RS 232, Adaptive Ethernet \\
& Interface \\
Immunity to Noise and Interference & Yes \\
Reading Range & $0-60 \mathrm{~m}$ \\
Operating Temperature & $-20^{\circ} \mathrm{C}$ to $60^{\circ} \mathrm{C}$ \\
Dimension & $190 \mathrm{~mm} \times 120 \mathrm{~mm} \times 40 \mathrm{~mm}$ \\
Output Power & $+15 \mathrm{dbm}$ \\
\hline
\end{tabular}


Table 2. Technical specification of active RFID tags used in this work.

\begin{tabular}{ll}
\hline \hline Parameter & Specifications \\
\hline Tag type & Wrist band and card type \\
Operating Frequency & $2.4-2.8 \mathrm{GHz}$ \\
UID (Unique Identification Number) & 4 Bytes \\
Output Power & $-6 \mathrm{dBm}$ \\
Battery Life & 3 Year \\
Reading Distance & $0-80$ Meters \\
\hline
\end{tabular}

\subsection{MRI System and CT scan}

Of the several imaging techniques available including ultrasound, X-rays, and CT scan, MRI is considered to be superior in several aspects. It is a non-invasive procedure [23], does not require any contrasting agents, is userfriendly and can provide three-dimensional images with good tissue contrasting. The MRI scanner used in this proposed work is an active shielded $0.3 \mathrm{~T} / 12.7 \mathrm{MHz}$ system (AIRIS Vento III, HITACHI, JAPAN) with an open bore system. The attractive feature of this MRI scanner is its ability to perform numerous imaging sequences that are exclusively associated with high-field scanners. The CT scanner used in this work was made by General Electric, USA.

\section{Methods}

\subsection{Functional Evaluation Test for RFID Tags}

Ten samples (five wristbands and five card tags) of RFID tags (shown in Fig. 2) were comprehensively evaluated to determine their functional alterations. Each tag was verified for its functionality before being exposed to the MRI examination. All the tags were mounted on non-conductive plastic sheet (dimension $24 \mathrm{~cm} \times 13 \mathrm{~cm} \times$ $10 \mathrm{~cm}$ ) in three different orientations (sagittal, axial and coronal), which is similar to the one expected during regular patient examination. The RFID reader was excluded from the evaluation process. The entire examination

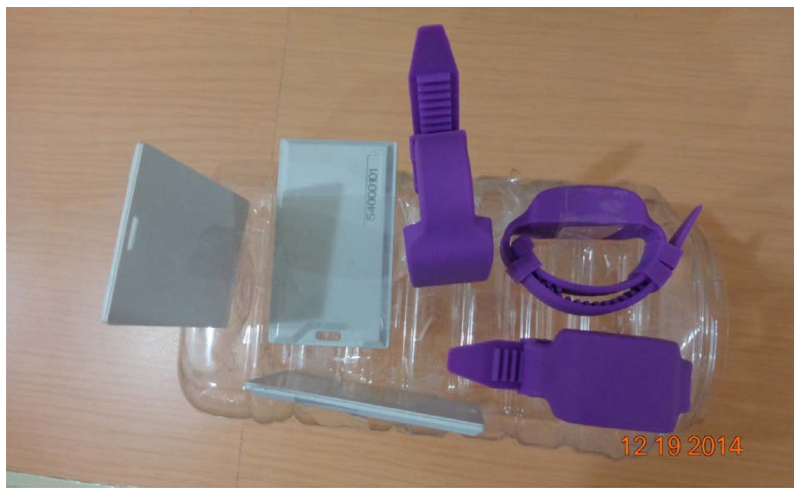

Fig. 2. (Color online) (White color-Card tags and Pink ColorWrist bands).

was carried out using cylindrical MRI phantom containing nickel solution. The plastic sheet containing RFID tags was affixed on the phantom by using glue. During the initial setup, we found that wristband tags, which are bulgier than card tags, were attracted towards the magnet when the phantom was moved closer to the magnet. No such effects occurred with the card tags, even if theyare moved closer to the magnet. Thus, we excluded wristband tags from our test and continued with card tags only. With the use of transmit-receive body radio frequency (RF) coil, a certain number of MRI sequences listed in Table 3, which are representative of MRI sequences performed during regular patient examination, were performed on each RFID tag successively for an average duration of 5 minutes per sequence. After thorough exposure to the radiation, the tags were taken outside the MRI room and assessed for functional evaluation.

The correct functioning of a RFID tag was verified using the following criteria: i) ability to read the UID of each tag accurately [16]; ii) ability to perform proper read and write operations on each tag; iii) ability to retain the contents or data that already stored in the memory; and iv) sustained no physical damage during the MRI examination.

Table 3. MRI sequences performed on each tag at $0.3 \mathrm{~T}$ for assessing tag's functionality.

\begin{tabular}{|c|c|c|c|c|c|c|}
\hline Pulse Séquence & T1-FSE & T1-GE & T2-FSE & T2-FSE & T1-SE & T2-SE \\
\hline \multicolumn{7}{|l|}{ Image Conditions } \\
\hline $\mathrm{TR}(\mathrm{ms})$ & 550 & 350 & 505 & 230 & 300 & 600 \\
\hline $\mathrm{TE}(\mathrm{ms})$ & 15 & 15 & 15 & 125 & 18 & 250 \\
\hline Flip Angle & $90^{\circ}$ & $90^{\circ}$ & $90^{\circ}$ & $90^{\circ}$ & $90^{\circ}$ & $90^{\circ}$ \\
\hline Field of View $(\mathrm{cm})$ & 38 & 34 & 35 & 31 & 26 & 26 \\
\hline Section Thickness (mm) & 5 & 5 & 5 & 5 & 6 & 6 \\
\hline Imaging Plane & sagittal & coronal & coronal & axial & sagittal & axial \\
\hline
\end{tabular}

(T1-SE, T1-Weighted Spin Echo; T1-GE, T1-Gradient Echo, T2-SE, T2-Weighted Spin Echo; T1-FSE, T1-Weighted Fast Spin Echo; T2-FSE, T2 Weighted Fast Spin Echo; TR-Recovery Time; TE-Echo Time) 
Table 4. MRI sequences performed on each tag at $0.3 \mathrm{~T}$ for image artifacts.

\begin{tabular}{lcc}
\hline \hline Pulse Séquence & T1-FSE & T1-GE \\
\hline Image Conditions & & \\
TR (ms) & 350 & 350 \\
TE (ms) & 15 & 15 \\
Flip Angle & $90^{\circ}$ & $90^{\circ}$ \\
Field of View (cm) & 34 & 34 \\
Section Thickness (mm) & 5 & 5 \\
Imaging Plane & coronal & coronal \\
\hline
\end{tabular}

(T1-FSE, T1-Weighted Fast Spin Echo; T1-GE, T1-Gradient Echo; TR-Recovery time; TE-Echo time)

\subsection{Assessment of MRI Artifacts}

In order to evaluate the imaging artifacts qualitatively, the imaging of the right wrist of the human volunteer was performed in two different settings. Informed consent was obtained from the volunteer as well as local ethics committee of the hospital. In the first setting, the image of right wrist was captured when no tags were attached on any part of the body. In the second setting, the image of the right wrist was captured when the tag was attached on it. For each case, the MRI sequences listed in Table 4 were performed to capture the images. For qualitative analysis, we compared the images captured with RFID tag on the right wrist with images captured without any RFID tags. Based on the level of severity, the artifacts were categorized into no artifact, mild artifact, moderate artifact and strong artifact.

\subsection{CT Scanning of RFID tags}

The CT images of the right wrist of the volunteer with and without the RFID tags (both wrist bands and card tags) attached were captured using CT technique of 80 $\mathrm{mAs}$ at $120 \mathrm{kV}$ for the duration of $60 \mathrm{~s}$.

\subsection{Assessment of RFID reader performance}

It was assumed that a uniform and symmetrical field exists outside the MRI scan room as done in other studies $[17,20]$. We positioned RFID reader one meter away from the copper shield separating the MRI scanner area and the patient waiting room. With the help of a tripod stand and a protractor, the RFID reader can be fixed at any desirable angle. The objectives of this particular test was to find out whether the following factors have any impact on the performance of the RFID reader stationed outside the MRI scan room: i) on/off of the MRI machine; ii) angle of the RFID reader; and iii) the type of RFID tags. The test was conducted with the following steps.

1. When the MRI machine was turned on, the patients

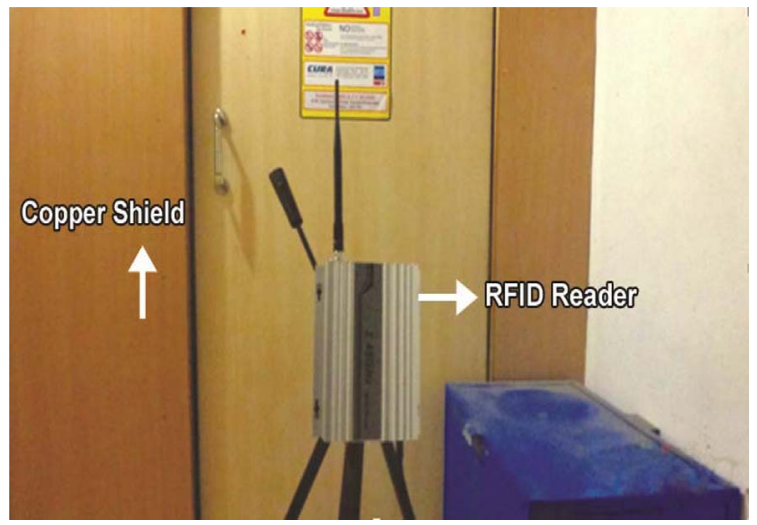

Fig. 3. (Color online) RFID reader positioned at $0^{\circ}$ angle.

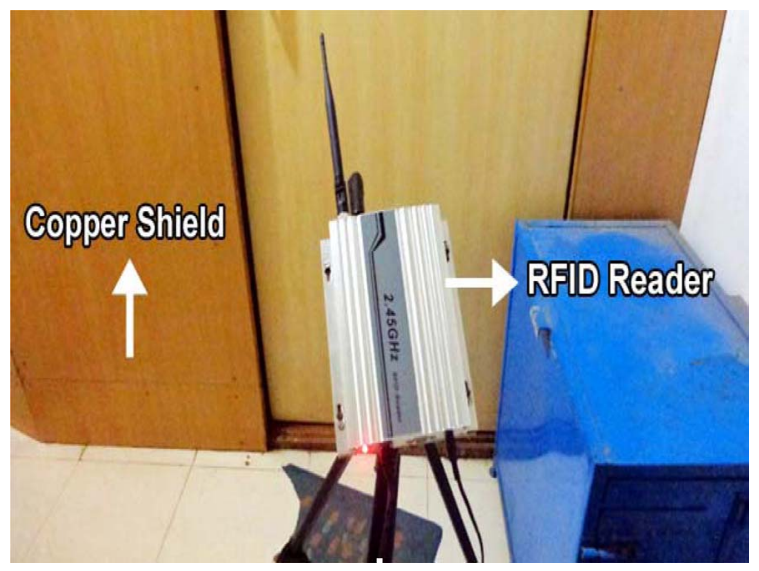

Fig. 4. (Color online) RFID reader positioned at $45^{\circ}$ angle.

with RFID tags attached on the wrist were requested to enter the waiting room, where the RFID reader was located. The distance between the RFID reader and the patient entry point was about $4.3 \mathrm{~m}$. There was only one possible direction of entry to the waiting room, and it was opposite to the position of the RFID reader. Initially, the reader was kept at $0^{\circ}$

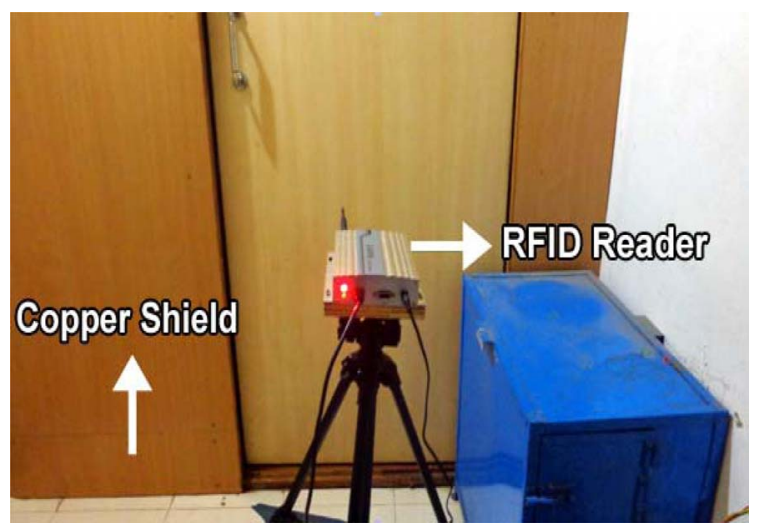

Fig. 5. (Color online) RFID reader positioned at $90^{\circ}$ angle. 
angle (vertical position as shown in Fig. 3). When the patient with the RFID tags entered the room, we noted down the maximum distance at which the RFID tag was detected, and the corresponding number of tag responses sent to the reader. This step was repeated for several times to confirm the reproducibility of the results. A similar set of procedures were followed when the reader was positioned at $45^{\circ}$ and $90^{\circ}$ angles (shown in Fig. 4 and Fig. 5).

2. When the MRI machine was turned off, the same procedures as in Step 1 were repeated, and the results were noted down.

\section{Results}

After the MRI examination, it was found that none of the card tags sustained any physical damage nor did they exhibit any malfunction. The reader was able to read the UID of each tag and perform read/write operations on each tag. The results also indicate that the contents stored in the memory of each tag can be retained. Overall, the results indicate that the functionality of the tags remains unchanged.

It was found that severe artifacts were present in the MRI images of the volunteer with RFID tag attached to the right wrist when the area of imaging is closer to the RFID tags. A visible signal loss or void was present in the images, and no useful information could be gathered from those images. The MRI images of the volunteer with and without the RFID tags attached to right wrist are shown in Fig. 6, Fig. 7 and Fig. 8 .

After CT scan, the functionality of both wristbands and card tags remain unaffected. But the quality of CT images was affected due to the presence of both types of RFID tags when the area of imaging was closer to RFID tags.

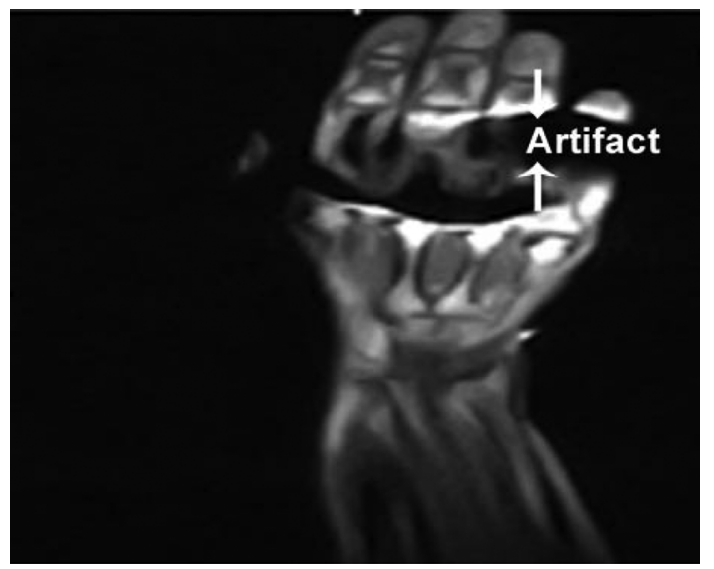

Fig. 6. MR Image of wrist of volunteer with RFID tag (Imaging sequence: T1-FSE; Imaging Plane-Coronal).

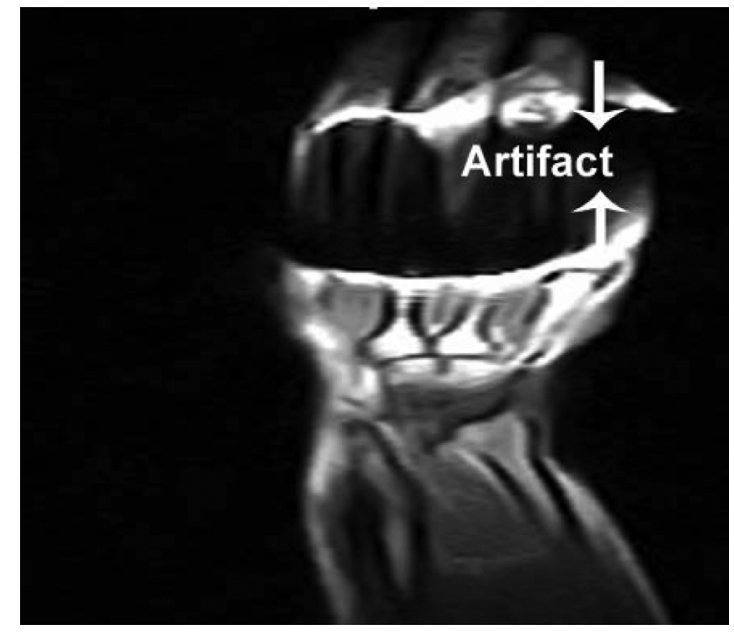

Fig. 7. MR Image of wrist of volunteer with RFID tag (Imaging sequence: T1-GE; Imaging Plane-Coronal).

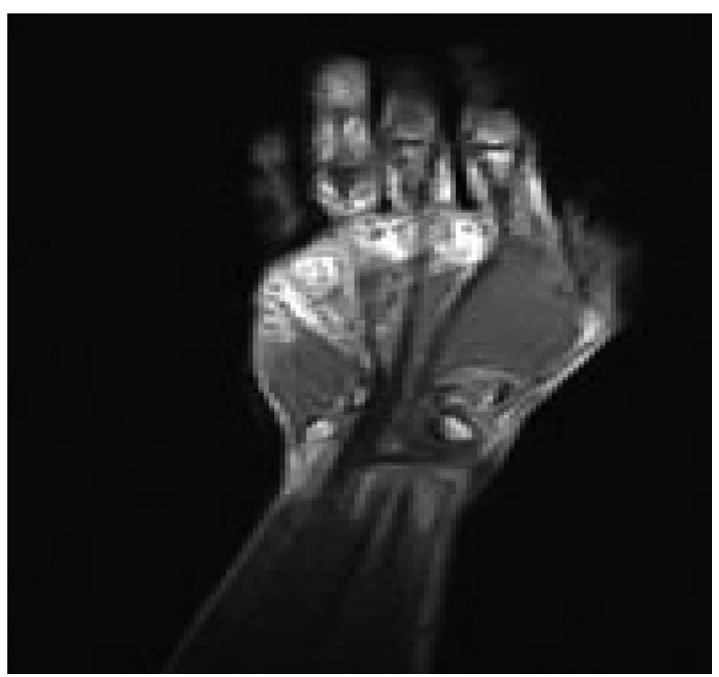

Fig. 8. MR Image of wrist of volunteer without RFID tag (Imaging sequence: T1-FSE; Imaging Plane-Coronal).

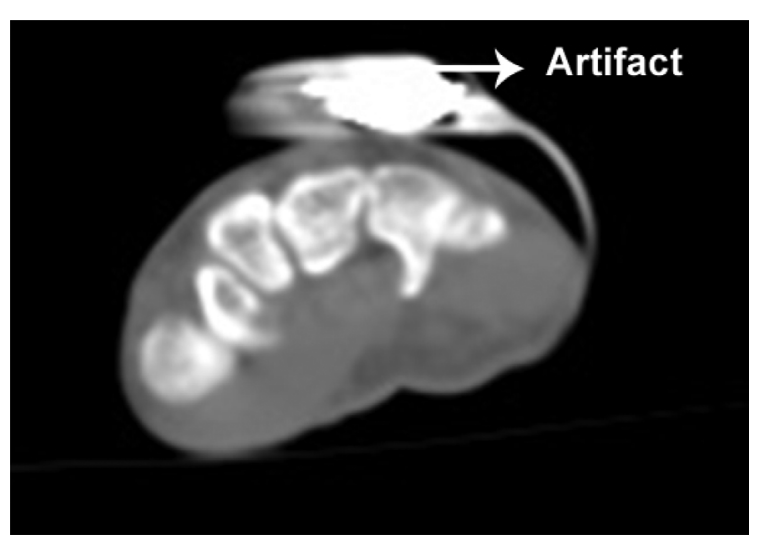

Fig. 9. CT Image of wrist of volunteer with RFID tag (CT imaging technique: $80 \mathrm{mAs}$ at $120 \mathrm{kV}$ ). 
However, if the area of imaging was far from the location of the RFID tags, the quality of images remained intact. The CT image of the volunteer with RFID tag attached to the right wrist is shown in Fig. 9.

The reader's performance was not affected during on/ off of the MRI machine, types of tags as well as the angle of the reader. The reader's performance was constant throughout the test.

\section{Discussion}

In this study, the functional alteration and imaging artifacts issues related to the use of active RFID tags attached to patients in $0.3 \mathrm{~T}$ MRI and CT systems was investigated. During initial experimental setup, wristband tags were excluded from the study due to their displacement towards the magnet when they were placed closer to the magnet. Wristband tags contained larger nickel batteries than the card tags and that could have been the reason for their displacement. We found that the functionalities of card tags remain unchanged after exposure to MRI examination. Although functional alteration of active $2.4 \mathrm{GHz}$ RFID tags was not reported under 1.5 $T$ MRI scanners, we need such investigation for lower field systems because of the complexities involved in the design of MRI scanners [24]. We found severe artifacts in the captured MRI when the region of interest (ROI) was closer to the RFID tags. Several factors, including static magnetic field of the scanner, orientation of the device, size of the device, magnetic susceptibility of the material and imaging sequences performed, can influence the artifacts in the images. Our results showed that the size of the card tags and the magnetic susceptibility of nickel battery resting inside the tags could extend the severity of the artifacts. However, the severity of the artifacts can be reduced by placing the RFID tags farther away from the ROI as well as by avoiding certain MRI sequences. A summary of different research works undertaken with respect to RFID in MRI is given in Table 5. From the table, we found that, RFID tags are safe to use in MRI environment irrespective of magnetic field strength of scanners and type of RFID tags used except in one study [18]. The only concern is the presence of artifacts in the images that has been captured in many similar studies. Also, it is observed from the table that, except proposed work and a study by [30], all other studies are involved with the implementation of passive RFID system which induces either small quantum of artifacts in the captured MRI images or not. The artifacts in these MRI images did not affect the diagnostic process by doctors. At the same juncture, the artifacts appeared in the MRI images of proposed work and a study by [30], which involved with implementation of an active RFID system, did affect the diagnostic process by doctors, if the ROI is closer to RFID tags.

Our study also proved that the on/off condition of the MRI machine, angle of the reader and the type of thugs did not affect the performance of the RFID reader stationed outside the MRI room. Moreover, our results also indicated that the EM interference between the MRI scanner and the RFID reader was not problematic. Furthermore, it was found that the RFID reader can be fixed at any point and any angle and that any card can be used for our convenience. As far as CT scan was concerned, the strong presence of nickel batteries inside the tags prevented them from getting damaged due to X-rays of CT scan. At the same juncture, the presence of those nickel batteries could be the reason for presence of strong artifacts in the $\mathrm{CT}$ images.

There are a few limitations of our study. This study involved only one MRI scanner that emitted a static magnetic field strength of $0.3 \mathrm{~T}$ as well as one CT scanner. Furthermore, only five tags were used for the exposure to the MRI field and ten tags for X-rays of CT scan. Even though active RFIDs are available in several frequencies, we restricted our application to RFID tags working at 2.5

Table 5. Summary of various research works undertaken.

\begin{tabular}{|c|c|c|c|c|}
\hline Author & RFID Used & $\begin{array}{l}\text { Magnetic Field of } \\
\text { MRI Scanner }\end{array}$ & $\begin{array}{c}\text { Is there any functional } \\
\text { change in tag? }\end{array}$ & $\begin{array}{c}\text { Is there any artifact } \\
\text { in MR images? }\end{array}$ \\
\hline Steffen et al. [15] & Passive, $13.56 \mathrm{MHz}$ & $1.5 \mathrm{~T}$ and $3.0 \mathrm{~T}$ & No & Yes \\
\hline Titterington et al. [16] & Passive, $129.0-133.2 \mathrm{KHz}$ and $135.2-139.4 \mathrm{KHz}$ & $1.5 \mathrm{~T}$ and $3.0 \mathrm{~T}$ & No & Yes \\
\hline Cheng et al. [26] & Passive, $915 \mathrm{MHz}$ & $1.5 \mathrm{~T}$ & No & Not evaluated \\
\hline JLamberg [27] & Verichip ${ }^{\mathrm{TM}}$ Human Implant & $1.5 \mathrm{~T}$ & Yes & Yes \\
\hline Periyasamy et al. [28] & Passive, $13.56 \mathrm{MHz}$ & $0.3 \mathrm{~T}$ & No & No \\
\hline Periyasamy et al. [29] & Passive, $920 \mathrm{MHz}$ & $0.3 \mathrm{~T}$ & No & No \\
\hline Fei et al. [30] & Active, $2.5 \mathrm{GHz}$ & $1.5 \mathrm{~T}$ & No & Yes \\
\hline Proposed Work & Active, $2.5 \mathrm{GHz}$ (Card tags only) & $0.3 \mathrm{~T}$ & No & Yes \\
\hline
\end{tabular}


GHz. The experimental procedure was limited to certain types of imaging sequences for functional analysis and imaging artifacts assessment in MRI and CT environment. The limitations exposed in this study can overcome by deploying this same active RFID in other field MRI scanners (either lower or higher magnetic field) with more number of RFID tags and experimenting with different types of MRI sequences.

\section{Conclusion}

Based on the clinical results obtained, we can conclude that only card type active RFID tags operating at $2.5 \mathrm{GHz}$ are suitable for patient identification purpose in $0.3 \mathrm{~T}$ MRI environment and both wristbands as well as card tags were suitable for the same purpose in CT environment. Careful consideration must be given in both MRI and $\mathrm{CT}$ environments if the area of imaging is closer to RFID tags. No external factors affected the performance of active RFID reader stationed outside the MRI scan room

\section{References}

[1] F. G. Shellock, J. Magn. Reson. Imaging 16, 721 (2002).

[2] F. G. Shellock and J. V. Crues, Radiol. 232, 635 (2004).

[3] T. O. Woods, J. Magn. Reson. Imaging 26, 1186 (2007).

[4] F. G. Shellock and A. Spinazzi, Am. J. Roentgenol. 191, 1 (2008).

[5] F. G. Shellock, T. O. Woods, and J. V. Crues, Radiol. 253, 26 (2009).

[6] K. Ohashi, S. Ota, L. O. Machado, and H. Tanaka, Comput. Biol. Med. 40, 545 (2010).

[7] M. Cavalleri, R. Morstabilini, and G. Reni, Conf. Proc. IEEE Eng. Med. Biol. Soc. 5, 3278 (2004).
[8] C. C. Liu, C. H. Chang, M. C. Su, H. T. Chu, S. H. Hung, J. M. Wong, and P. C. Wang, Comput. Meth. Prog. Bio. 104, 435 (2011).

[9] P. Peris-Lopez, A. Orfila, A. Mitrokotsa, and J. C. Van der Lubbe, Int. J. Med. Informs. 80, 13 (2011).

[10] E. Etchells, C. O. Neill, and M. Bernstein, World J. Surg. 27, 936 (2003)

[11] G. Calcagnini, F. Censi, M. Maffia, L. Mainetti, E. Mattei, L. Patrono, and E. Urso, IEEE Trans. Inf. Technol. Biomed. 16, 1051 (2012).

[12] A. Oztekin, F. M. Pajouh, D. Delen, and L. K. Swim, Decis. Support. Syst. 49, 100 (2010).

[13] W. Zhou and S. Piramuthu, Decis. Support. Syst. 50, 222 (2010).

[14] E. W. T. Ngai, J. K. L. Poon, F. F. C. Suk, and C. C. Ng, Inf. Syst. Front. 11, 405 (2009).

[15] T. Steffen, R. Luechinger, S. Wildermuth, C. Kern, C. Fretz, J. Lange, and F. H. Hetzer, Patient. Saf. Surg. 4, 1 (2010).

[16] B. Titterington and F. G. Shellock, Magn. Reson. Imaging 31, 1439 (2013).

[17] C. Y. Cheng and J. W. Chai, J. Med. Syst. 36, 3423 (2012).

[18] J. Lamberg, http://www.rfidjournal.com. Accessed July 12, 2012.

[19] M. Periyasamy and R. Dhanasekaran, Scientific World Journal, 1 (2014).

[20] M. Periyasamy and R. Dhanasekaran, Biomed. Eng.-App. Bas. C 26, 1 (2014).

[21] X. Fei, S. Lei, S. Gao, L. Wei, and L. Wang, Biomed. Eng. Online 13, 1 (2014).

[22] Fiocchi Serena, Parazzini Marta, Paglialonga Alessia, and Ravazzani Paolo, Bioelectromagnetics 32, 408 (2011).

[23] N. R. Jagannathan, Biophys. 36, 341 (1999).

[24] M. A. Baker and I. Macdonald, Vet. Radiol. Ultrasoun. 52, 161 (2011). 